\title{
Diabetes and menopause
}

Volume 6 Issue 4 - 2016

\section{Introduction}

Menopause is an important transition in women's reproductive life, as it signals the end of fertility. The timing of menopause is also an important determinant of future disease risk. An early age at menopause is associated with an increased risk of cardiovascular disease (CVD) and decreased incidence of cancers. ${ }^{1}$ Menopause is defined as amenorrhea for at least 12months and Type 2 diabetes mellitus is defined as FBS $\geq 126 \mathrm{mg} / \mathrm{dl}$, 2-hr post prandial blood glucose level $\geq 200 \mathrm{mg} / \mathrm{dl}$ and $\mathrm{HbA} 1 \mathrm{C} \geq 6.5{ }^{2}$

Normoglycemia will minimize or prevent many of the complications of diabetes, including blindness, renal disease, neuropathy, bone fractures and peripheral vascular disease. However, being female does contribute an added risk for gender-related problems. The ages and stages of a diabetic woman's life bring increased risk at every milestone, despite exquisite attention to glucose control.

\section{Type I diabetes and menopause}

Individuals with type 1 diabetes experience accelerated senescence Therefore, one would expect other indicators of biological age, such as the occurrence of the menopause transition, to begin prematurely among women with type 1 diabetes. ${ }^{3}$ The mean age at natural menopause is statistically significantly for type 1 diabetic women compared with nondiabetic sisters or control subjects $(41.6,49.9$, and 48.0 years, respectively, $P=0.05$ ). This results in a 6 -year reduction in the number of reproductive years $(30.0,37.0$, and 35.2 years, respectively, $P=0.05$ ) for women with type 1 diabetes. An earlier menopause, results in a $17 \%$ decrease in reproductive years. With an older age at menarche and younger age at menopause, type 1 diabetic women had an average of 6 fewer reproductive years than their sisters or control subjects. ${ }^{4}$

Glycosylation of functional proteins may cause ovarian dysfunction or type 1 diabetes. Autoimmune mechanisms can also be linked to menopausal age. Loss of ovarian function and subsequent decline in endogenous estrogens, can explain these differences in risk. It is observed that oophorectomized women have less favorable glucose and insulin levels that may explain the link between premature menopause and diabetes risk. ${ }^{5}$

Another explanation for an early menopause among type 1 diabetic women may be related to prolonged hyperglycemia and/or other long-term complications of the disease. In addition, peripheral hyperinsulinemia and insulin resistance occurs among approximately one-half of individuals with type 1 diabetes. ${ }^{6}$ Hyperinsulinemia is associated with the polycystic ovarian syndrome (PCOS) and is characterized by hyperandrogenemia and amenorrhea. ${ }^{7}$ Because insulin and androgen levels are highly correlated in women with PCOS, one may speculate that the young age at menopause in women with type 1 diabetes may be mediated, in part, through peripheral hyperinsulinemia and/or hyperandrogenemia. However, the occurrence of PCOS in women with type 1 diabetes has rarely been reported. ${ }^{8}$ Thus, factors unrelated to long-term diabetes may also be important determinants of the menopause transition.

\author{
Vijayalakshmi Chandrasekhar \\ Assistant Professor (OBGY), GITAM Institute of Medical \\ Sciences \& Research, India
}

Correspondence: Vijayalakshmi Chandrasekhar, Assistant Professor (OBGY), GITAM Institute of Medical Sciences \& Research, Rushikonda,Visakhapatnam, India,

Emailsvijaya2003@yahoo.com

Received: November 29, 2016 | Published: December 13, 2016

\section{Type II diabetes \& menopause}

Type II diabetes, also known as insulin-resistant diabetes mellitus, is the most common form of diabetes. Type 2 diabetes mellitus is a heterogeneous group of disorders characterized by variable degrees of insulin resistance, impaired insulin secretion, and increased glucose production. In type II diabetes the patient's body either cannot produce insulin or cannot effectively use the insulin it produces. There are many reasons why women over forty are most likely to be diagnosed with type II diabetes. Lower levels of the hormones estrogen and progesterone, and human growth hormone contribute to lower metabolism and obesity which is the major cause of type II diabetes. ${ }^{9}$

\section{Autoimmunity}

Approximately $20-40 \%$ of women with premature ovarian failure also have autoimmune disorders, particularly autoimmune thyroid disease. ${ }^{10}$ In addition, circulating antiovarian autoantibodies have been observed with a greater frequency among subjects who experienced premature ovarian failure compared with healthy control subjects, even though they had no evidence of overt autoimmune disease. Thus, there appears to be a strong positive association between autoimmunity and premature menopause. Given the well-documented associations between the HLA region of chromosome 6 and type 1 diabetes risk, the genetic factors that increase the risk of autoimmune disorders may also influence age of menopause. It has been shown that HLA-linked genes contribute to the levels of sex hormones in men and age at menarche in women. In addition, associations between premature ovarian failure and HLA-DR3 and HLA-DR4 which confer susceptibility to type 1 diabetes, have been observed. ${ }^{11}$ Time to menopause was associated with the DR4-DQA $1 * 0301-\mathrm{DQB} 1 * 0302$ haplotype but not the DR3-DQA1*0501-DQB1*0201 haplotype. ${ }^{4}$

\section{Complications of menopausal diabetes}

Infections: Patients with type 2 diabetes mellitus are at increased risk of infections, with the urinary tract being the most frequent infection site. ${ }^{12-15}$ Urinary tract infections are more common, more 
severe, and carry worse outcomes in patients with type 2 diabetes mellitus. They are also more often caused by resistant pathogens. Various impairments in the immune system, poor metabolic control, and incomplete bladder emptying due to autonomic neuropathy may all contribute to the enhanced risk of urinary tract infections in these patients Diabetes under pharmacologic treatment is associated with increased risk of clinically apparent UTI in postmenopausal women. The spectrum of UTI in these patients ranges from asymptomatic bacteriuria (ASB) to lower UTI (cystitis), pyelonephritis, and severe urosepsis. Serious complications of UTI, such as emphysematous cystitis and pyelonephritis, renal abscesses and renal papillary necrosis, are all encountered more frequently in type 2 diabetes than in the general population. ${ }^{16,17}$ Pyelonephritis was found to be 4.1 times more frequent in pre-menopausal diabetic women than in women without diabetes. ${ }^{18,19}$ There is no indication to treat diabetic patients with asymptomatic bacteriuria.

\section{Osteoporosis}

Osteoporosis is the most common metabolic disorder, which is accompanied by a decrease in bone mass and destruction of bone tissue. In addition to disability, it increases mortality among individuals who suffer from osteoporosis related fractures. Osteoporosis is characterized by a simultaneous decrease in bone mineral density and bone matrix. As a result, the bone mass decreases but its composition remains intact.

Osteoporosis and diabetes mellitus are major and growing public health problems particularly among postmenopausal women. T score of the lumbar vertebra (L2-L4) and femoral neck are measured with Dual Energy X-ray Absorptiometry (DXA). According to World Health Organization definition T-score $\leq-2 / 5$ SD less than mean BMD of young adults is considered as osteoporosis, T-score between zero to -1 is considered as normal BMD and T-score between -1 to $-2 / 5$ considered as osteopenia, and at last T-score $\leq-2.5$ plus a history of fracture, considered as a severe established osteoporosis. ${ }^{20}$

Estrogen deficiency is correlated with a rapid reduction in bone mineral density. As a result, women who have had shorter than average exposure to estrogen during their life (late onset of menarche, early menopause, longer than 6 months cessation of menstrual cycle are at higher risk of osteoporosis. Estrogen blocks the absorbing activity of osteoclasts, enhances the trans-intestine transportation of calcium, increases the absorption of calcium from kidneys and protects the osteoclasts. But after menopause, because there is a lack of ovarian function and estrogen, the activity of osteoclasts and the pace of bone destruction increases, which will result in $25-30 \%$ destruction in bone mass during a 5-10 years period. A high rate of bone destruction is seen in individuals with chronic hyperglycemia, with the prevalence of fractures in hip, arm and leg is higher in old aged diabetic women compared to the non-diabetic ones. Bone loss is more common in those with $\mathrm{HbA} 1 \mathrm{C} \geq 7$ compare to those with HbA1C $<7$. Based on lumbar T-scores, $44 \%$ of diabetic and $43.3 \%$ of non-diabetic cases have osteopenia and $39 \%$ of diabetic and $40 \%$ of non-diabetic cases have osteoporosis. Based on femoral neck T-score, osteopenia is found in $47.5 \%$ of diabetic and $27.3 \%$ of non-diabetic cases, and osteoporosis in $4.9 \%$ of diabetic and $4.5 \%$ of non-diabetic cases. $^{21} 50 \%$ of men and $70 \%$ of women older than 70 , suffer from osteoporosis or osteopenia. Proper control of this risk factor can be an effective way in prevention of osteoporosis.

\section{Cardiovascular disease}

Postmenopausal type II diabetic women are also at higher risk for cardiovascular disease and osteoporosis. Postmenopausal women experience more type 2 diabetes and cardiovascular diseases than their premenopausal counterparts. After menopause there is an increase in dyslipidemia, especially reduced levels of HDL. In addition to HDL changes, menopause is also accompanied by changes in apolipoprotein (apo) A and (apo) B, fibrinogen, and hyperinsulinemia. Accelerated thickening of muscle basement capillary membrane and stiffening of connective tissue generally occur in nondiabetic subjects $>50$ years of age but are commonly observed among young adults with type 1 diabetes, particularly those with long disease duration. ${ }^{22}$

The risk for developing heart disease in a diabetic patient is two to three times higher than the risk for patients without diabetes. The morbidity of cardiovascular disease dramatically increases after menopause. In such cases, estrogen depletion has been speculated as a cause of the disease, as estrogen plays an anti-atherogenic role both in vivo and in vitro. Although hormone replacement therapy was not reported to prevent cardiovascular disease in clinical trials, this was due to the increased frequency of thrombosis produced by estrogen in advanced atherosclerosis and to the adverse effect of co prescribed progesterone. The fact that females are known to live several years longer than males world-wide strongly supports the anti atherogenic effect of estrogen. ${ }^{23}$

Premenopausal type 1 diabetic women are at high risk of developing cardiovascular disease. Therefore, an earlier menopause transition may exacerbate their likelihood of developing these complications during their postmenopausal years. The etiology of diabetic atherosclerosis is due to the decrease of Nitric Oxide (NO) bioavailability as a result of high glucose levels. The incidence of cardiovascular diseases is increased in elderly diabetic women. NO is synthesized by nitric oxide synthetase enzyme. This utilizes L-arginine as a substrate and produces L-citrulline as the second reaction product. L-arginine can be synthesized from L-citrulline in endothelial cells through a recycling pathway. This pathway may be the principal mechanism for sustaining localized L-arginine availability for endothelial nitric oxide synthase (eNOS)-catalyzed NO production. This is impaired in diabetic women. ${ }^{24}$

Postmenopausal women with diabetes have increased dyslipidemia compared with nondiabetic women. Among diabetic women, current users of HRT had significant different lipid and glucose control levels than those who never used HRT. Diabetic and non diabetic postmenopausal women currently taking HRT had better lipoprotein profile than never or previous users of HRT. Diabetic women currently taking HRT had better glycemic control than never or previous users of HRT. ${ }^{25}$

The use of hormone replacement therapy (HRT) is seen to be associated with a better cardiovascular health profile in healthy postmenopausal women. HRT may be effective in lowering the risk of cardiovascular disease in healthy menopausal women, and although postmenopausal HRT is recommended to prevent disease and prolong life, little data are available to examine the association between HRT and emerging hyperlipidemic indicators separately for diabetic subjects and nondiabetic postmenopausal women. Favorable lipid and glucose profiles are observed among HRT users with lower fasting glucose, GHb, total cholesterol, and non-HDL in this group of diabetic postmenopausal women. ${ }^{26-28}$

Fibrinogen is an important component in platelet aggregation and is also a risk indicator for cardiovascular disease, including coronary heart disease, stroke, and peripheral artery disease, and has been closely linked to smoking, hypertension, and total cholesterol. Fibrinogen is significantly lower among current HRT users than women who never used HRT for both diabetic and nondiabetic women. Similar findings 
are observed in postmenopausal women aged 52-65 years, where HRT usage is associated with significantly lower fibrinogen concentration and decreased plasma viscosity. ${ }^{29}$

$C$-reactive protein is an important inflammatory biomarker that has been suggested to have an intermediary role in the pathogenesis of cardiovascular disease in individuals with type 2 diabetes. Post menopausal diabetic women have a higher level of C-reactive protein. Both men currently using HRT had higher levels of C-reactive protein than previous or never users. ${ }^{30}$

\section{Conclusion}

The subtle changes in body composition that occurs during menopausal transition negatively influences glucose metabolism. Risk factors and complications of diabetes in perimenopausal women is significantly higher than non diabetic women. There is, as yet, no cure for diabetes, but new research has developed new ways to monitor and regularize blood sugar. Managing diabetes whilst going through menopause is a twin challenge for most women. Avoiding obesity, maintaining a tight control over blood sugar levels with drugs and physical activity and judicious use of HRT minimizes the added risks for diabetic women at climacteric.

\section{Acknowledgments}

None.

\section{Conflicts of interest}

The authors declare there are no conflicts of interest.

\section{Funding}

None.

\section{References}

1. Monninkhof EM, van der Schouw YT, Peeters PH. Early age atmenopause and breast cancer: are leaner women more protected? A prospective analysis of the Dutch DOM cohort. Breast Cancer Res Treat. 1999;55(3):285-291.

2. Incalzi RA, Corsonello A, Pedone $\mathrm{C}$, et al. Identifying older diabetic patients at risk of poor glycemic control. BMC Geriatr. 2002;2:4.

3. Verzola D, Gandolfo MT, Gaetani G. Accelerated senescence in the kidneys of patients with type 2 diabetic nephropathy. Am J Physiol Renal Physiol. 2008;295(5):1563-1730.

4. Dorma JS, Steenkiste AR, Foley TP, et al. Menopause in Type 1 Diabetic Women. Diabetes. 2001;50(8):1857-1862.

5. Kritz-Silverstein, Barrett Connor E, Wingard DL. Hysterectomy, oophorectomy and Heart Disease Risk I Older Women. Am J Pub Health. 1997;87(4):676-680.

6. Wilcox G. Insulin and insulin resistance. Clin Biochem Rev. 2005;26(2):19-39.

7. Sheehan MT. Polycystic Ovarian Syndrome. Clin Med Res. 2004;2(1):13-27.

8. Hector F, Morreale E, Martin NBR. Type 1 Diabetes and Polycystic Ovary Syndrome: Systematic Review and Meta-analysis Diabetes Care. 2016;39(4):639-648.

9. Lizcano F, Guzman G. Estrogen deficiency and the origin of obesity during menopause. BioMed Research International. 2014;11.

10. FarageMA, Miller KW, Maibach I. Effects of Menopause on Autoimmune Diseases. Expert Rev of Obstet Gynecol. 2012;7(6):557-571.
11. Anasti JN, Adams S, Kimzey LM, et al. Karyotypically normal spontaneous premature ovarian failure: evaluation of association with the class II major histocompatibility complex. J Clin Endocrinol Metab. 1994;78(3):722-723.

12. Patterson JE, Andriole VT. Bacterial urinary tract infections in diabetes. Infect Dis Clin North Am. 1997;11(3):735-750.

13. Joshi N, Caputo GM, Weitekamp MR, et al. Infections in patients with diabetes mellitus. N Engl J Med. 1999;341(25):1906-1912.

14. Boyko EJ, Fihn SD, Scholes D, et al. Risk of urinary tract infection and asymptomatic bacteriuria among diabetic and nondiabetic postmenopausal women. Am J Epidemiol. 2005;161(6):557-564.

15. Shah BR, Hux JE. Quantifying the risk of infectious diseases for people with diabetes. Diabetes Care. 2003;26(2):510-513.

16. Kofteridis DP, Papadimitraki E, Mantadakis E, et al. Effect of diabetes mellitus on the clinical and microbiological features of hospitalized elderly patients with acute pyelonephritis. $J$ Am Geriatr Soc. 2009;57(11):2125-2128

17. Mnif MF, Kamoun M, Kacem FH, et al. Complicated urinary tract infections associated with diabetes mellitus: pathogenesis, diagnosis and management. Indian J Endocrinol Metab. 2013;17(3):442-445.

18. Scholes D, Hooton TM, Roberts PL, et al. Risk factors associated with acute pyelonephritis in healthy women. Ann Intern Med. 2005; 142(1):20-27.

19. Boyko EJ, Fihn SD, Scholes D, et al. (2002) Diabetes and the risk of acute urinary tract infection among postmenopausal women. Diabetes Care. 2002;25(10):1778-1783.

20. Yamamoto M, Yamaguchi M, Yamauchi M, et al. Serum pentosidine levels are positively associated with the presence of vertebral fraucture in post menopausal women with type 2 diabetes mellitus. J Clin Endocr Metab. 2008;93(2):1013-1019.

21. Karimifar M, Pasha MA, Salari A, et al. Evaluation of bone loss in diabetic postmenopausal women. J Res Med Sci. 2012;17(11):10331038 .

22. Kilo C, Vogler NL, Williamson JR. Muscle capillary basemen membrane changes related to aging and to diabetes mellitus. Diabetes. 1972;21(8):881-898.

23. Holm P, Heidi LA, Anderson MR, et al. The Direct Antiatherogenic Effect of Estrogen. Circulation. 1999;100:1727-1733.

24. Hoshiyama M, Li B, Yao J, et al. Effect of high glucose on nitric oxide production and endothelial nitric oxide synthase protein expression in human glomerular endothelial cells. Nephron Exp Nephrol. 2003;95(2):62-68

25. Fuyong D, Virtue E, Wang $\mathrm{H}$, et al. Metabolomic analyses for atherosclerosis, diabetes, and obesity. Biomark Res. 2013;1:17-20.

26. Ferrara A, Karter AJ, Ackerson LM, et al. Hormone Replacement Therapy Is Associated With Better Glycemic Control in Women With Type 2 Diabetes. Diabetes Care. 2001;24(7):1144-1150.

27. Crespo CJ, Smit E, Snelling A, et al. Hormone Replacement Therapy and Its Relationship to Lipid and Glucose Metabolism in Diabetic and Nondiabetic Postmenopausal Women Results from the Third National Health and Nutrition Examination Survey (NHANES III). Diabetes Care. 2002;25(10):1675-1680

28. Schnatz PF, Schnatz JD. Dyslipidemia in menopause: mechanisms and management. Obstet Gynecol Surv. 2006;61(9):608-613.

29. Lee AJ, Lowe GD, Smith WC, et al. Plasma fibrinogen: its relationship with oral contraception, the menopause, and hormone replacement therapy. Clin Biochem 1992;25(5):403-405.

30. Bowden DW, Lohman K, Hsu FC, et al. Hormone replacement therapy is associated with increased C-reactive protein in women with Type 2 diabetes in the Diabetes Heart Study. Diabet Med. 2006;23(7):763-767. 